\title{
Genome evolution driven by host adaptations results in a more virulent and antimicrobial-resistant Streptococcus pneumoniae
} serotype I 4

\author{
Feng Ding ${ }^{\dagger 1,2}$, Petrus Tang ${ }^{\dagger 3}$, Mei-Hua $\mathrm{Hsu}^{4}$, Peng Cui ${ }^{1,2}$, Songnian $\mathrm{Hu}^{1}$, \\ Jun $\mathrm{Yu}^{1}$ and Cheng-Hsun Chiu*4
}

\author{
Address: ${ }^{1}$ The CAS Key Laboratory of Genome Sciences and Information, Beijing Institute of Genomics, Chinese Academy of Sciences, 100029 \\ Beijing, PR China, ${ }^{2}$ Graduate School of the Chinese Academy of Sciences, Chinese Academy of Sciences, 100029 Beijing, PR China, ${ }^{3}$ Department \\ of Parasitology, Chang Gung University College of Medicine, Kweishan 333, Taoyuan, Taiwan and ${ }^{4}$ Department of Pediatrics, Chang Gung \\ Children's Hospital, Chang Gung University College of Medicine, Kweishan 333, Taoyuan, Taiwan \\ Email: Feng Ding - dingfeng@big.ac.cn; Petrus Tang - petang@mail.cgu.edu.tw; Mei-Hua Hsu - mei.huw@gmail.com; \\ Peng Cui - cuipeng@big.ac.cn; Songnian Hu - husn@genomics.org.cn; Jun Yu - junyu@big.ac.cn; Cheng- \\ Hsun Chiu* - chchiu@adm.cgmh.org.tw \\ * Corresponding author †Equal contributors
}

Published: 13 April 2009

BMC Genomics 2009, 10:158 doi:10.1186/147|-2164-10-158
Received: 22 July 2008

Accepted: 13 April 2009

This article is available from: http://www.biomedcentral.com//47/-2/64//10/158

(c) 2009 Ding et al; licensee BioMed Central Ltd.

This is an Open Access article distributed under the terms of the Creative Commons Attribution License (http://creativecommons.org/licenses/by/2.0), which permits unrestricted use, distribution, and reproduction in any medium, provided the original work is properly cited.

\begin{abstract}
Background: Streptococcus pneumoniae serotype 14 is one of the most common pneumococcal serotypes that cause invasive pneumococcal diseases worldwide. Serotype 14 often expresses resistance to a variety of antimicrobial agents, resulting in difficulties in treatment. To gain insight into the evolution of virulence and antimicrobial resistance traits in S. pneumoniae from the genome level, we sequenced the entire genome of a serotype 14 isolate (CGSPI4), and carried out comprehensive comparison with other pneumococcal genomes. Multiple serotype 14 clinical isolates were also genotyped by multilocus sequence typing (MLST).

Results: Comparative genomic analysis revealed that the CGSPI 4 acquired a number of new genes by horizontal gene transfer (HGT), most of which were associated with virulence and antimicrobial resistance and clustered in mobile genetic elements. The most remarkable feature is the acquisition of two conjugative transposons and one resistance island encoding eight resistance genes. Results of MLST suggested that the major driving force for the genome evolution is the environmental drug pressure.

Conclusion: The genome sequence of $S$. pneumoniae serotype 14 shows a bacterium with rapid adaptations to its lifecycle in human community. These include a versatile genome content, with a wide range of mobile elements, and chromosomal rearrangement; the latter re-balanced the genome after events of HGT.
\end{abstract}




\section{Background}

Streptococcus pneumoniae is a major human respiratory pathogen that causes a variety of serious infections such as pneumonia, otitis media, meningitis and hemolytic uremic syndrome (HUS). It is estimated that each year more than 1 million deaths are attributed to $S$. pneumoniae infection worldwide [1]. Furthermore, since 1990 antimicrobial resistance has been escalating in S. pneumoniae [2], resulting in difficulties in the treatment of pneumococcal infections. Antimicrobial resistance of $S$. pneumoniae is associated with increasing incidence of invasive pneumococcal diseases in children as well as clinical failures of antimicrobial treatment [3]. Although the 7-valent conjugate vaccine has been shown highly efficacious in the prevention of invasive diseases caused by vaccine serotypes $[4]$, the emergence of non-vaccine serotype infections has occurred [5], bringing new challenges to the prevention of pneumococcal diseases.

HUS is a rare but severe complication of infectious diseases. The majority of HUS cases are associated with enterohemorrhagic Escherichia coli; however, HUS associated with invasive S. pneumoniae infection has been increasingly reported over the years, always with a high mortality and long-term morbidity [6]. Among the vaccine-preventable serotypes of $S$. pneumoniae, serotype 14 is often invasive, as evidenced by its high predilection to cause necrotizing pneumonia and other devastating complications, including HUS $[7,8]$. In the pre-vaccine era, serotype 14 was one of the most common serotypes of $S$. pneumoniae that caused invasive pneumococcal diseases worldwide [9-11]. Moreover, serotype 14 often expresses resistance to a variety of antimicrobial agents, including penicillin, erythromycin, and ceftriaxone. To date, four complete genome sequences of $S$. pneumoniae are available in public database, including two laboratory strains (TIGR4 and D39), one avirulent strain (R6) and one multidrug-resistant strain (Spn23F) [12-15]. Furthermore, a total of twelve draft sequences have been published recently $[16,17]$. These pneumococcal genomes provide us a good opportunity to undertake comprehensive comparative studies on the virulence and antimicrobial resistance mechanisms of this microorganism. We described here the features of $S$. pneumoniae serotype 14 genome and the changes of the genome structure and contents compared with other pneumococcal genomes. The diversity and dynamics of the distributed genome of $S$. pneumoniae serotype 14 , especially the mobile genetic elements carrying a number of virulence genes and antimicrobial resistance determinants, are highlighted.

\section{Results \\ General genome features}

The single circular chromosome of strain CGSP14 contains 2,209,198 bp with a $\mathrm{G}+\mathrm{C}$ content of $39.5 \%$ (Figure

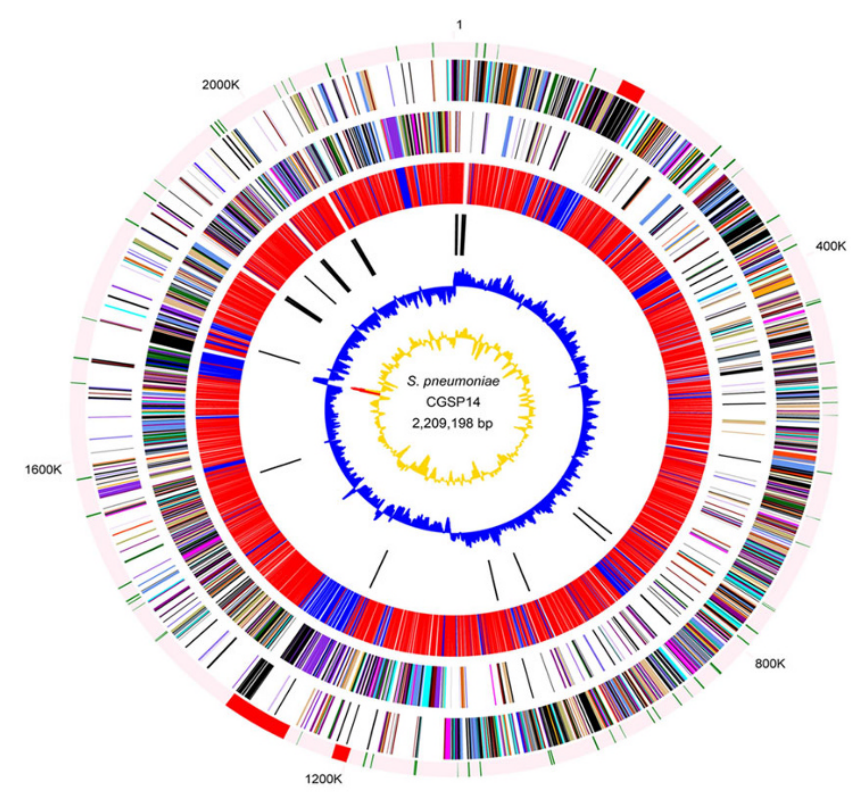

Figure I

Circular representation of S. pneumoniae chromosome. Outer circle: distribution of mobile genetic elements (red, transposons and putative resistance islands; green, IS elements). Second and third circles: predicted ORFs on the plus and minus strand, respectively. ORFs are distinguished by different colors in the COG classification http:// www.ncbi.nlm.nih.gov/COG. Fourth circle: conserved genes (red) of the four S. pneumoniae genomes and accessory elements (blue). Fifth circle: tRNA and rRNA genes. Sixth circle: GC skew. Seventh circle: GC content.

$1)$. The sequence of the genome has been deposited in the GenBank database (accession no. CP001033). Base pair one of this chromosome was assigned within the putative origin of replication. The genome has 58 tRNAs, 12 rRNAs and 3 structural RNAs, including 4 rRNA operons. Biological roles were assigned to $67 \%$ of the 2,206 predicted protein-coding sequences (CDSs), according to the classification scheme adapted from Riley [18]. Seventynine percent of the coding sequences were transcribed in the same orientation as DNA replication, a feature that appears to be common in other low GC Gram-positive bacteria [15]. The replication termination site is localized near 1.1 megabase pairs by GC skew analysis. This region is located almost exactly opposite the origin of replication on the chromosome (Figure 1). The genome includes 65 pseudogenes, the majority of which are IS elements and hypothetical proteins.

Previous studies showed that the S. pneumoniae genome is rich in IS elements, which make up more of the genome than of any other bacterial genomes sequenced to date $[13,15]$. In the CGSP14 genome we identified 80 IS elements (Additional file 1). The majority of the IS elements 
appeared to be degenerate due to insertions, deletions, or point mutations, and only twelve were intact in CGSP14 genome. Although these degenerate IS elements might be inactive and non-functional, they could provide the potential sites for homologous recombination to acquire novel genes from related species.

\section{Comparative genomic analysis}

Comparative analysis of CGSP14 genome with four complete genomes and twelve draft pneumococcal genomes (Table 1) provided new insights into the rapid evolution of the pneumococcal genome. S. pneumoniae, as with other bacterial pathogens, possesses a conserved core genome with interspersing regions of small and large scale differences (Figure 2). In total, 1,619 orthologous genes were shared by the seventeen pneumococcal genomes (Additional file 2). By searching the orthologous genes against COG database, $36 \%$ were found to be metabolism-related, 36\% were associated with other known functions, $16 \%$ had poorly characterized functions, and $12 \%$ had no hits in the COG database and encoded mainly hypothetical proteins (Additional file 3). In addition, we found CGSP14 shared the largest number of orthologous genes (2055 and 2049 respectively) with strains Spn23F and SPnINV200 among the sixteen strains used for comparative genomic analysis, indicating that the CGSP14 genome shows highest homology to the two sequenced strains.

The genes on the distributed genomes were further analyzed. Alignment analysis revealed that at least eight distributed clusters were present in CGSP14 genome; most of the genes were related to virulence or antimicrobial resistance. These include a lantibiotic synthesis gene cluster, the capsular locus, a large cell wall surface anchor protein, two transposons, a resistance island, a possible phage remnant and a gene cluster with unknown functions. Meanwhile, we displayed the genome-wide GC content in Figure 3. All the eight clusters had deviated GC content, suggesting they could be recent acquisitions through horizontal gene transfer (HGT) in CGSP14. The distribution of the eight gene clusters among the seventeen pneumococcal genomes was shown in Table 2. Particularly, the two conjugative transposons were found to be unique in the CGSP14 genome.

Alignment analysis indicated that chromosomal rearrangements occurred in S. pneumoniae (Figure 2). Compared with other published pneumococcal genomes, chromosomal inversions were identified in CGSP14 genome. A 189-kb inversion occurred across the replication termination site (from 1,010 kb to $1,199 \mathrm{~kb}$ ). Chromosomal inversion across the replication axis usually is believed to rebalance the unbalanced chromosomal architecture caused by the insertion of large DNA segments [19]. In the CGSP14 genome, we found that most of the acquired-DNA segments (totally $80.5 \mathrm{~kb}$ ), mainly composed of transposons and IS elements, resided in left of the replication axis (Figure 1 ). These observations suggested that the integration of transposons and IS elements affected the balance of the chromosomal architecture. This imbalance might cause the chromosomal inversion in CGSP14. This inversion led to transfer of 25 genes from the left to the right of the replication axis. Besides, a 19-kb inversion (from $832 \mathrm{~kb}$ to $851 \mathrm{~kb}$ ) was observed in CGSP14 relative to TIGR4, G54, and INV200, while the gene order in this 19-kb segment is consistent to INV200. The gene cluster is not intact in other pneumococcal genomes. Further analysis showed that the four rearrangement breakpoints were located within the IS elements. Through chromosomal rearrangements, S. pneumoniae evolved to maintain genome stability after HGT that

Table I: Sixteen published genomes of $S$. pneumoniae used for comparative genomic analysis

\begin{tabular}{|c|c|c|c|}
\hline Strain & Accession number & Serotype & Sequencing center \\
\hline TIGR4 & AE005672 & 4 & TIGR \\
\hline R6 & AE007317 & 2 & Eli Lilly and Company \\
\hline D39 & CP000410 & 2 & TIGR \\
\hline SP3-BS7I & AAZZZ00000000 & 3 & Center for Genomic Sciences \\
\hline SP6-BS73 & ABAA00000000 & 6 & Center for Genomic Sciences \\
\hline SP9-BS68 & $\mathrm{ABAB} 00000000$ & 9 & Center for Genomic Sciences \\
\hline SPII-BS70 & $\mathrm{ABAC} 00000000$ & 11 & Center for Genomic Sciences \\
\hline SPI4-BS69 & ABAD00000000 & 14 & Center for Genomic Sciences \\
\hline SPI8-BS74 & ABAE 00000000 & 6 & Center for Genomic Sciences \\
\hline SPI9-BS75 & ABAF00000000 & 19 & Center for Genomic Sciences \\
\hline SP23-BS72 & ABAG00000000 & 23 & Center for Genomic Sciences \\
\hline G54 & CP001015 & $19 \mathrm{~F}$ & TIGR \\
\hline Spn23F & FM211187 & $23 \mathrm{~F}$ & Sanger \\
\hline SPnINVI04B & - & 1 & Sanger \\
\hline SPnINV200 & - & 14 & Sanger \\
\hline SPnOXCI4I & - & 3 & Sanger \\
\hline
\end{tabular}




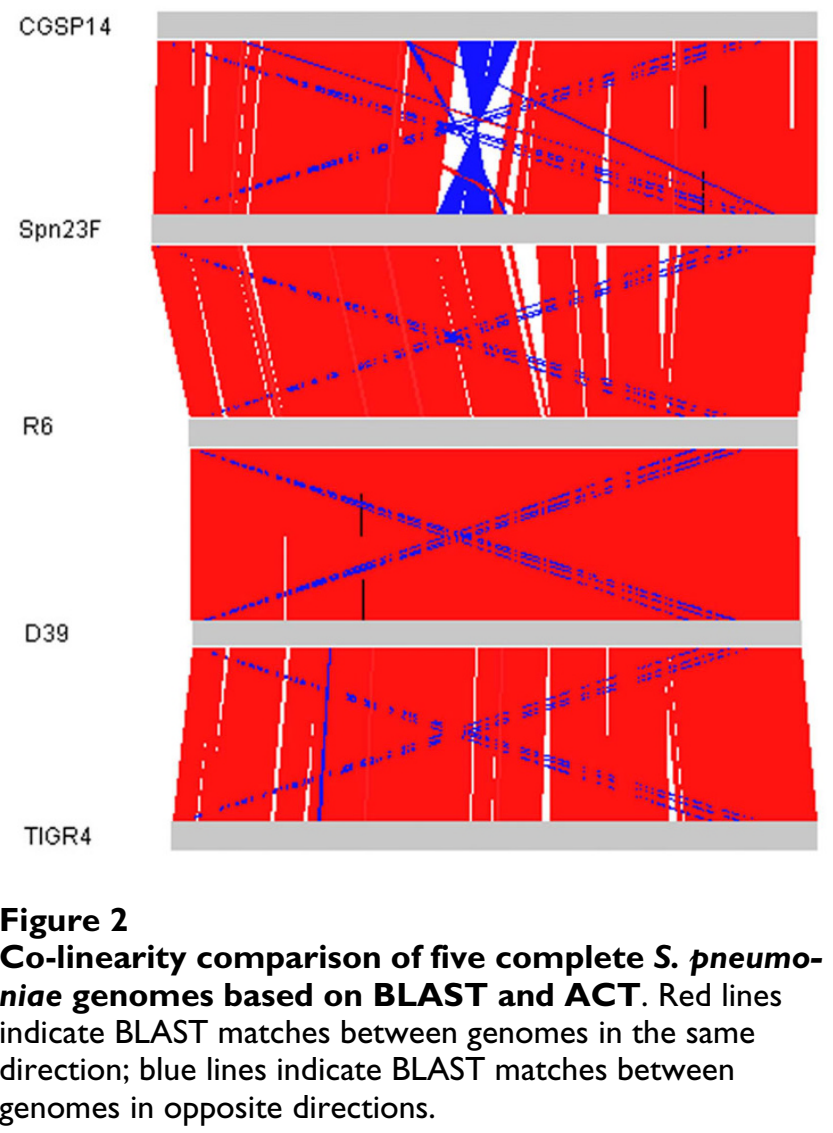

might confer genes necessary for the organism to survive or replicate in its environmental niche.

\section{Antimicrobial Resistance genes}

CGSP14 is resistant to a variety of antimicrobial agents. The antimicrobial resistance determinants among the seventeen penumococcal strains were compared and listed in Additional file 4. CGSP14 contained 18 antimicrobial resistance determinants, while the number of antimicrobial resistance determinants in other strains varied from 9 to 12 . Nearly half of the antimicrobial resistance determinants in CGSP14 were associated with mobile genetic elements.
The genome contained two large conjugative transoposons, which were found as composite elements of the known transposons. The first one containing 69 open reading frames (ORFs), was a 68 -kb conjugative transposon (Figure 4A). Since this transposon had never been described previously, we named it Tn2008, a novel conjugative transposon. Sequence analysis indicated that Tn2008 was a composite of three transposons. A 50-kb DNA segment carrying chloramphenicol resistance gene (cat) could be an independent conjugative transposon and at left terminus of this transposon, two ORFs were designated as intergrase and relaxase required for transposition. The sequences of the ORFs within this transposon were highly homologous to those of $\mathrm{Tn} 5252$, which have been reported in $S$. pneumoniae before $[20,21]$. The Tn5252-like transposon was split into a $46-\mathrm{kb}$ proximal region and a 4-kb distal region after the insertion of a 13$\mathrm{kb}$ segment. The insertion appeared in the same position in Spn23F, which contained a 81-kb conjugative transposon [12]. The 13-kb insertion was identified as another independent transposon, which also owned the intergrase and excisionase at the right terminus for independent transposition; this transposon carried 3 genes coding for erythromycin, streptothricin and kanamycin resistance, a feature similar to the known Tn1545 [22]. Another 5-kb segment, as an insertion in the 13-kb transposon, resembled the transposon Tn917 [23], which contained 3 ORFs, encoding erythromycin resistance protein (ermB), resolvase and transposase. Overall, this novel conjugative transposon, a composite of three transposons, carried 5 antimicrobial resistance genes.

The other 23-kb conjugative transposon in CGSP14 contained 23 ORFs (Figure 4C). Sequence analysis demonstrated that this transposon was also a composite of two transposons. An 18-kb DNA segment carrying a tetracycline resistance gene (tet $M$ ) could be an independent transposon, which contained two ORFs encoding for intergrase and excisase; this transposon shows high similarity to the transposon Tn916 (Figure 4C) [24]. Another 5 -kb segment carrying an erythromycin resistance gene $(\mathrm{ermB})$, as an insertion, was identified as Tn917-like transposon. The structure of this composite transposon, i.e., a

Table 2: Distribution of the eight gene clusters among S. pneumoniae and other species of Streptococcus

\begin{tabular}{lll}
\hline Gene clusters & Strains of S. pneumoniae & Strains of other species (identity) \\
\hline Lantibiotic biosynthesis proteins & CGSPI4, SPnINV200 & S. thermophilus CNRZI066 (70-88\%) \\
Tn3872-like transposon & CGSPI4 & - \\
Capsular locus & CGSPI4, SPI4-BS69, SPnINV200 & - \\
19-kb insertion & CGSPI4, SPnINVI04B, SPnINV200, G54, TIGR4 & - \\
Possible phage remnant & CGSPI4, SP6-BS73, SPII-BS70, SPI4-BS69, SPI9-BS75, G54 & S. pyogenes MGAS6I80 (76-88\%) \\
Putative resistance island & CGSPI4, SP9-BS68, SPI4-BS69, SPI9-BS75, SPnINV200 & - \\
Tn2008 & CGSPI4 & - \\
Cell wall surface anchor protein & CGSPI4, TIGR4 & - \\
\hline
\end{tabular}




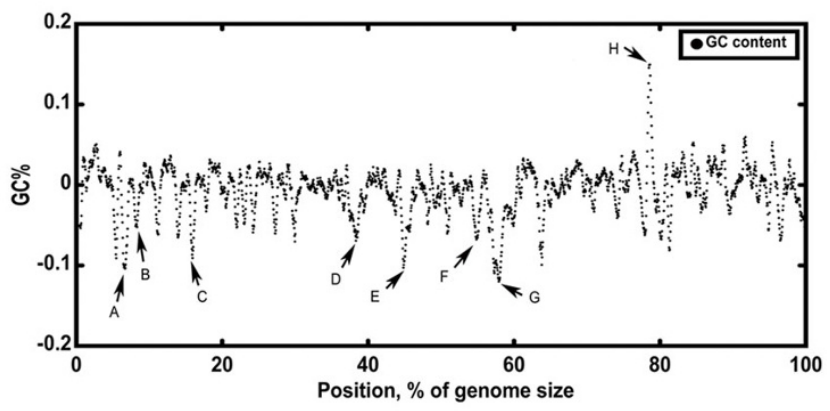

Figure 3

GC content of the CGSP I 4 chromosome. The GC content is calculated using the sliding-window technique ( window size $=10000 \mathrm{bp}$; shift size $=1000 \mathrm{bp}$ ). Zero of the $Y$ axis indicates the average GC content of the genome, which is $39.5 \%$. Black arrows indicate transposons and potential genomic islands in the S. pneumoniae genome. A, lantibiotic synthesis gene cluster; $\mathrm{B}, \mathrm{Tn} 3872$-like transposon; C, capsular locus; D, 19 kb inversion; $E$, possible phage remnant; F, putative resistance island; G, Tn2008; H, cell wall surface anchor family protein.

Tn917-like transposon inserted by a Tn916 transposon, resembled that of $\operatorname{Tn} 3872$. The structure of $\operatorname{Tn} 3872$ has been described in S. pneumoniae [25]; thus, this $23-\mathrm{kb}$ conjugative transposon was defined as a Tn3872-like transposon.

Among the seventeen published $S$. pneumoniae genomes, an 81-kb conjugative transposon and a $67-\mathrm{kb}$ conjugative transposon also appeared in the genomes of Spn23F and G54, respectively. The two conjugative transposons were both composed of a Tn916-like transposon and a Tn5252like transposon $[12,16]$, similar to Tn2008 in CGSP14. However, comparative analysis suggested that genetic variations occured among the three conjugative transposons (Figure 4B). In CGSP14 and Spn23F, the Tn5252-like transposons carry a chloramphenicol resistance gene, which seems missing in G54, and is replaced by an ABCtype antimicrobial peptide transport system. The Tn916like transposon in Spn23F carries a tetracycline resistance gene, and in G54, it carries a tetracycline resistance gene and an erythromycin resistance gene. In contrast, the Tn916-like element of Tn2008 in CGSP14 lost the locus encoding the tetracycline resistance gene, while a DNA segment encoding three antimicrobial resistance genes, a transcriptional repressor and a Tn917-like transposon was inserted into this position. The variation of antimicrobial resistance determinants in the three conjugative transposons showed that the conjugative transposons have experienced frequent recombination and deletion events after the Tn916-like element integrated into the larger conjugative transposon, probably due to different selective pressures.

In addition to the two conjugative transposons carrying antimicrobial resistance genes, we identified a $14.4-\mathrm{kb}$ genomic region (Figure 5), which appeared to be a resistance island in CGSP14. The 14.4-kb region carried a chloramphenicol resistance gene (cat) and a gene encoding methionyl-tRNA synthetase 2 (metS2). Further analysis showed that the island shared an average $\mathrm{G}+\mathrm{C}$ content of $33.6 \%$, much lower than the average of the genome $(39.5 \%)$. This island contained several genes associated with genome instability, including one site-specific recombinase and multiple IS elements which might be responsible for the lateral transfer of the genomic region. Furthermore, the associated ORFs had diverse phylogenetic origin (data not shown). Based on these features, we deemed the 14.4-kb region as a resistance island; to our knowledge, this was for the first time described in S. pneumoniae. This 14.4-kb resistance island was also seen in the draft genomes of CGSSp14BS69, CGSSp19BS75, CGSSp9BS68 and SPnINV200. BLAST results showed that the sequences in this island showed high identity to each other. The comparison between CGSP14 and SPnINV200 was demonstrated in Figure 4. Since this island carried two antimicrobial resistance genes, the presence of this resistance island may be associated with the increased multidrug resistance of these strains.

Distinct from the characterized antimicrobial resistance determinants associated with mobile genetic elements in CGSP14, there were several chromosome-encoded determinants that also contributed to antimicrobial resistance. These included a tellurite resistance protein (tehB), a bacitracin resistance protein $(b a c A)$, a cadmium resistance transporter $(c a d D)$, a multidrug resistance efflux pump $(m d t G)$, two $\beta$-lactam resistance factors $(f e m A B)$, and three metallo- $\beta$-lactamases.

\section{Penicillin-binding proteins}

Penicillin-resistant pneumococci are prevalent throughout the world. One mechanism conferring penicillin nonsusceptibility is alterations of penicillin-binding proteins (PBP). Alterations in PBP genes result in reduced affinity for penicillin and other $\beta$-lactams. Five high-molecularweight PBP genes ( $p b p 1 a, p b p 1 b, p b p 2 a, p b p 2 b, p b p 2 x$ ) dispersed in $S$. pneumoniae. Among the five genes, highly variable $p b p 2 x, p b p 1 a$, and $p b p 2 b$ are considered most important in antimicrobial resistance [26]. By allele assignments of the three PBP genes in the seventeen penumococcal genomes, CGSP14 had the most variable $p b p 2 x$, $p b p 1 a$, and $p b p 2 b$. Although the sequence variations of these PBP genes in CGSP14 differed from two other strains of serotype 14 (SPnINV200 and SP14-BS69), they 


\section{A}

$\left.\right|_{\text {Tn5252-like }} ^{\text {Tn916-like }}$

B

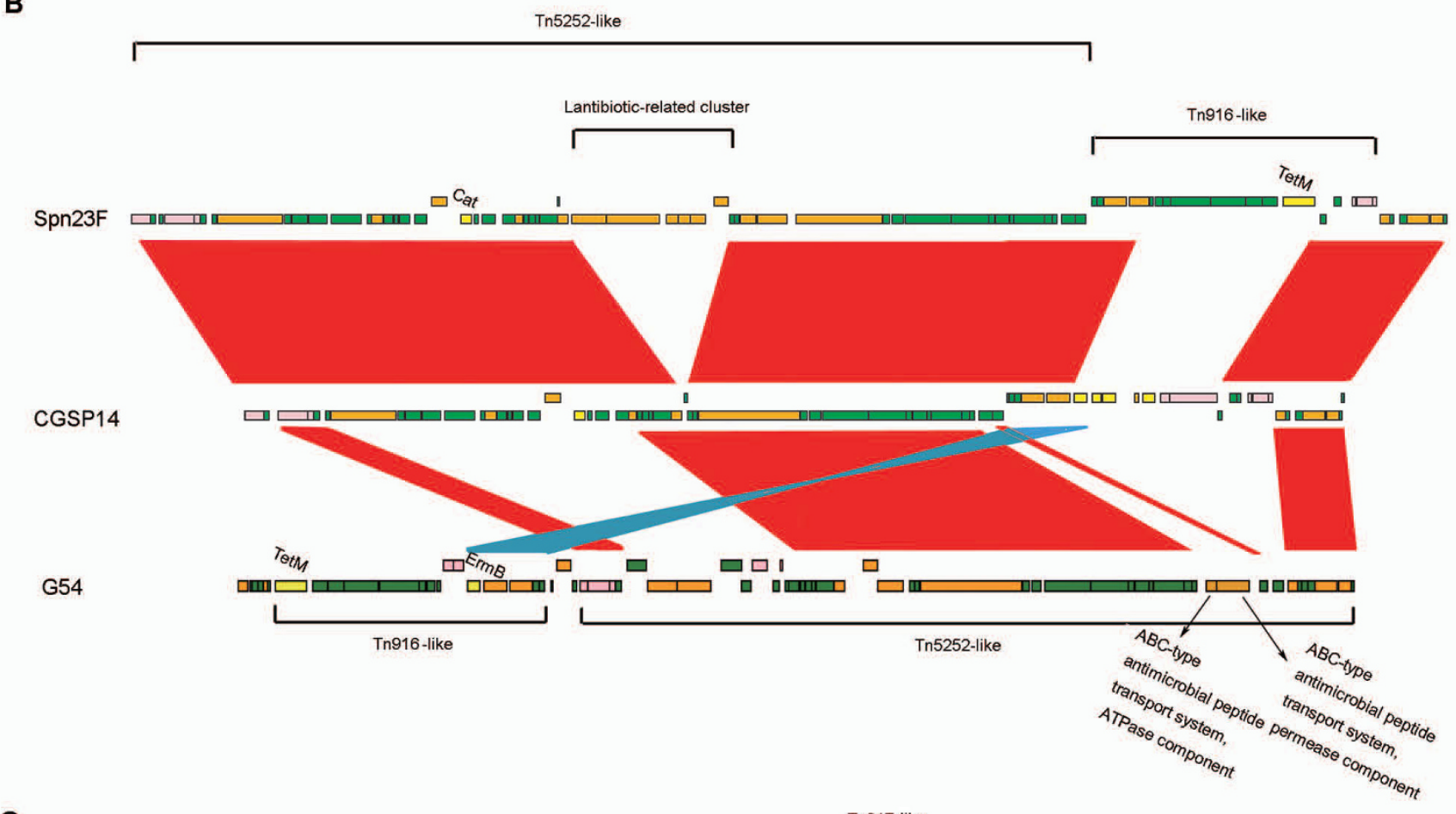

C

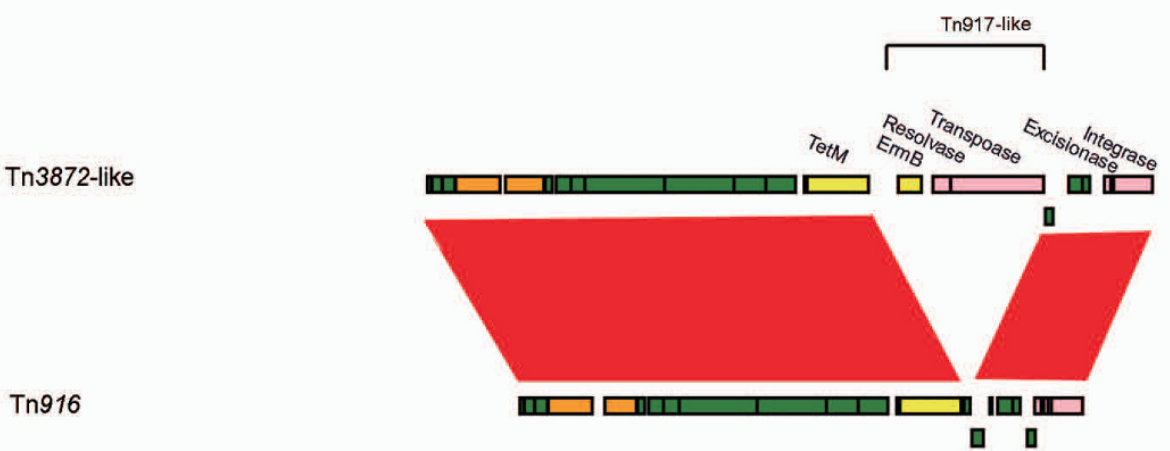

\section{Figure 4}

Representation of the two conjugative transpsons in CGSPI4. (A) Schematic portrayal of the 68-kb transposon in CGSPI4. Genes are coloured according to their putative functions. Pink boxes represent genes associated with mobility. Yellow boxes represent antimicrobial resistance genes. Orange boxes represent genes with other known functions. Green boxes represent hypothetical proteins. (B) Comparison of the conjugative transposons in the G54 and Spn23F genomes with CGSPI4. Red bands indicate BLAST matches between genomes in the same direction; blue bands indicate BLAST matches between genomes in opposite directions. (C) Schematic portrayal of the Tn3872-like transposon in CGSPI4 and comparison of this conjugative transposon with $\mathrm{Tn} 916$. The genes, tetM, ermB, cat, sat, and aph-3, confer tetracycline and minocycline resistance, erythromycin resistance, chloramphenicol resistance, streptothricin resistance, and kanamycin resistance, respectively. 


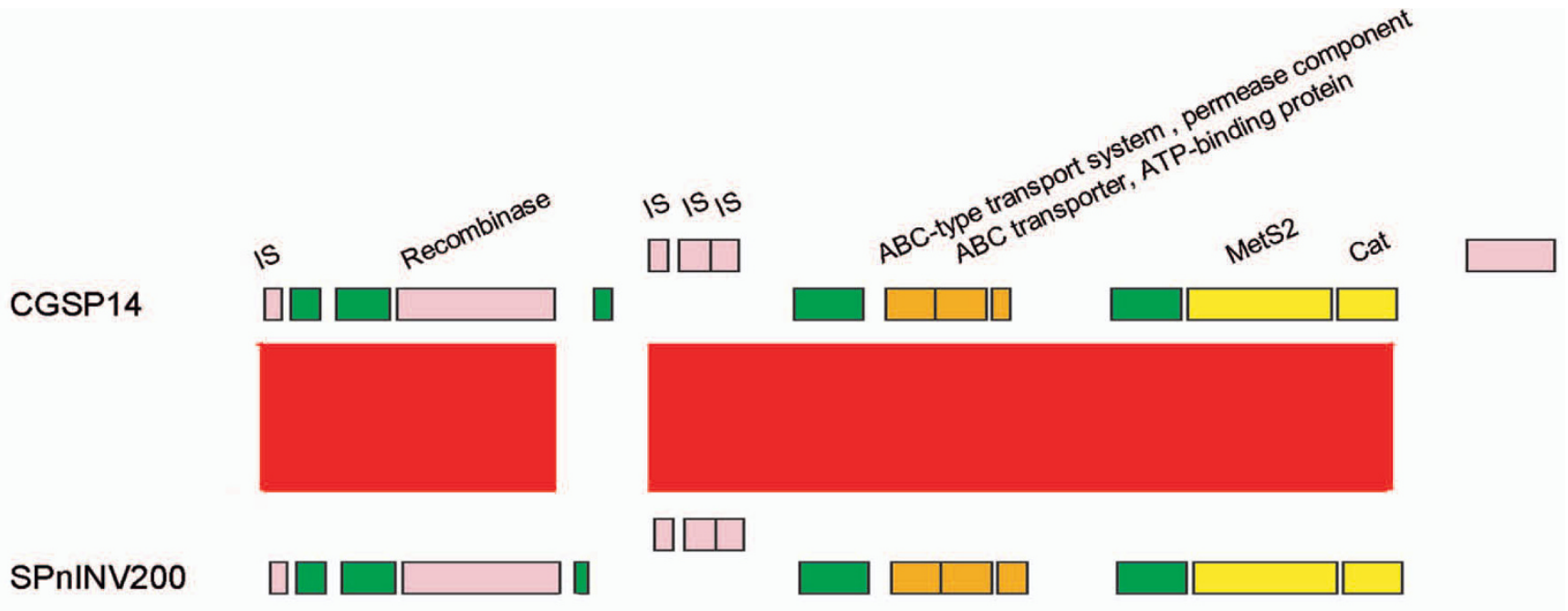

\section{Figure 5}

Comparison of the putative resistance island in CGSPI4 and SPnINV200. Genes are coloured as in Figure 4. Red bands indicate BLAST matches between genomes in the same direction.Cat confers chloramphenicol resistance. MetS2 encodes methionyl-tRNA synthetase 2 which confers high-level MetS inhibitors resistance.

were almost identical to those of the strain Spn23F, suggesting that $p b p 2 x, p b p 1 a$, and $p b p 2 b$ genes probably had gone through frequent homologous recombination between serotypes 14 and 23F.

\section{Virulence genes}

The polysaccharide capsule is the principal pneumococcal virulence determinant. $S$. pneumoniae are divided into 91 serotypes depending on different capsular structures. Studies suggested that certain serotypes have a greater potential to cause invasive disease than others $[27,28]$. The clinical isolates of $S$. pneumoniae in Asia are largely confined to a limited number of serotypes, namely $6 \mathrm{~B}, 9 \mathrm{~V}$, $14,19 \mathrm{~F}$, and 23F [29]. In CGSP14 genome, a 19.4-kb gene cluster (SPCG0345 to SPCG0363) was identified to be involved in the synthesis of the capsular polysaccharide, flanked by two IS elements on each side, either truncated or disrupted, which were remnants of IS1202 and IS1167, respectively. Compared to strain 34359 of serotype 14 and strain SPnINV200 for which the capsular locus was determined [30], the capsular locus of CGSP14 differed at 3 ' end (Additional file 5). The gene wciY was divided into two orfs (SPCG0358 and SPCG0359) in CGSP14. This gene was unique in serotype 14, but its function was unknown. However, a previous study showed that the disruption of this gene did not affect capsular production [31]. Besides, the orf (SPCG0360) immediately downstream of these two genes in CGSP14 was found to contain a deletion of 5 units of a 306-bp tandem repeat, compared with the corresponding genes in strain 34359 and SPnINV200. The gene belonged to the surface anchored protein family but its function also remained unclear. With the exception of these two genes, other genes in the capsular locus were almost identical among the three strains of serotype 14 [30]. As has been described [30], serotype 14 utilized the Wzx/Wzy-dependent pathway to synthesize their capsular polysaccharide (Additional file 6).

In addition to the capsule, $S$. pneumoniae produced a number of other virulence factors, such as pneumolysin, hydrogen peroxide and cell surface proteins [32]. According to how they are linked to the cell surface, the surface proteins of $S$. pneumoniae are divided into three families: choline-binding proteins, LPXTG-anchored proteins, and lipoproteins [32]. Surface proteins of CGSP14 based on computer prediction are shown in Additional file 7.

Several members of the choline-binding protein family are known to be important for virulence, including the autolysin $(l y t A)$, choline binding protein $\mathrm{A}(p s p C)$, and pneumococcal surface protein A ( $p s p A)$. PspC is involved in the adhesion of bacteria to the nasopharynx [33]. PspA is a highly variable protein and involved in inhibition of complement activation [32]. Choline binding protein PcpA is postulated to be an adhesin because it contains leucine-rich repeats [34]. The seventeen penumococcal genomes all harbored one copy of these virulence determinants, while CGSP14 and SP19-BS75 both obtained another copy of $p s p A$ and $p c p A$ due to a 7-kb-long DNA insertion adjacent to a remnant transposase. The $7 \mathrm{~kb}$ sequences in CGSP14 and SP19-BS75 showed high identity to each other. 
Proteins that contain the LPXTG amino acid motif are common in most Gram-positive bacteria. The LPXTG motif near to the carboxyl terminal of the protein is recognized and linked to the cell wall by a sortase enzyme [32]. Neuraminidase is one of the LPXTG-anchored proteins. Neuraminidase cleaves $\mathrm{N}$-acetylneuraminic acid from oligosaccharides, glycoproteins, glycolipids and is viewed as a virulence factor in microbial pathogenesis [32]. Analysis of the available genome sequences of $S$. pneumoniae indicated that this microorganism had at least three neuraminidases [13-15]. All the three neuraminidases are present in CGSP14. Both nanA and nanB are present in all the other sixteen penumococcal strains, while nan $C$ is present only in eight. The presence of nanC might be associated with the increased virulence of some strains of $S$. pneumoniae. Zinc metalloprotease is also a member of LPXTGanchored protein family. From the published genome sequences of $S$. pneumoniae, four zinc metalloproteases were discovered. CGSP14 contained three of them, including iga, $z m p B$ and $z m p D$. Zinc metalloproteinases belong to a group of hypervariable surface proteins, the hypervariability of these proteins are due to frequent HGT in these regions, enabling antigenic escape [35].

Besides these common virulence proteins, one unusual protein in LPXTG-anchored proteins family was found. The gene, SPCG1750, encoded a 4695-animo acid protein, containing 528 imperfect repeats of the amino acid motif SASASAST. This surface protein shows homology to SP1772 (4776-animo acid) in TIGR4. The surface protein is located in the vicinity of nine glycosyl transferases in CGSP14, all of which are present on a 40.5-kb segment flanked by two IS elements. The 40.5 -kb region seems to be an insertion in CGSP14 and TIGR4 due to HGT, as this region has not been found in other genomes.

Lantibiotics are peptide antibiotics with high antimicrobial activity against several Gram-positive bacteria. They are ribosomally synthesized and posttranslationally modified [36]. In CGSP14, we identified a 5.4-kb locus encoding three proteins related to lantibiotic biosynthesis: a lantibiotic dehydratase, a lantibiotic synthetase and a lan-

CGSP14
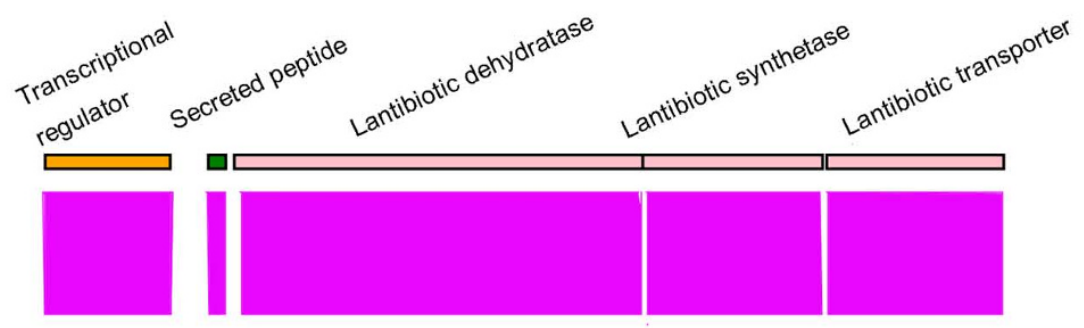

SPnINV200
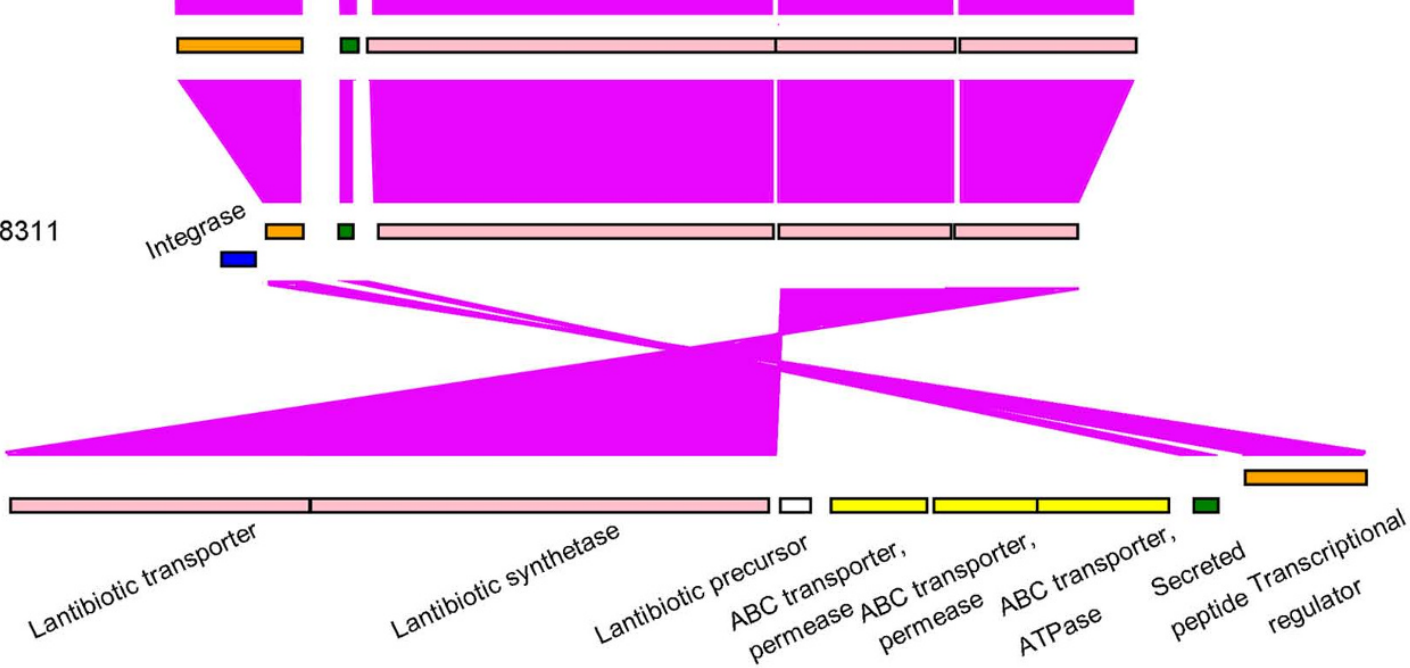

S. thermophilus LMG 18311

\section{Figure 6}

Comparison of the lantibiotic synthesis loci of CGSP I 4 and other genomes. Genes are represented by boxes coloured according to the gene key, with gene designations above or below each box. The genes encoded in this locus in the genomes of CGSPI4, SPnINV200 and S. thermophilus are highly homologous, but they have no sequence similarity to those in the genome of SPN23F. Purple bands correspond to genes that have similar function in the lantibiotic synthesis in each genome. 
tibiotic efflux protein, nearby a transcriptional regulator (Figure 6). By a thorough search against other sixteen genomes, this gene cluster shows high similarity to the corresponding locus in the genome of another serotype 14 strain SPnINV200. Recent studies reported that the strains SP23-BS72 and Spn23F of serotype 23 also contained lantibiotic synthesis gene clusters [12,37]; however, comparative analysis indicated that they showed no sequence similarity to those found in CGSP14 and SPnINV200. Furthermore, we performed a BLAST search against the nr database, and found the locus in the serotype 14 has a high similarity $(70 \%-88 \%$ identity) to that in Streptococcus thermophilus. Therefore, this locus in the serotype 14 might encode a new type of lantibiotic, different from those found in the serotype 23. This finding suggests that communication of virulence genes has occurred among different species of Streptococci.

\section{What drives the genome evolution?}

In this study, we further analyzed 20 clinical isolates of $S$. pneumoniae serotype 14 , all from sterile sites, by multilocus sequence typing (MLST), in addition to CGSP14, which belonged to ST15. The most common sequence type was ST876 (7 isolates), followed by ST13 (5) and ST46 (5) (Table 2). Only one ST15 was identified among the 20 isolates. ST876 and ST46 were prevalent in Taiwan, while both ST13 and ST15 belonged to variants of the international England ${ }^{14}$ clone (ST9). Capsular switching could have occurred between serotypes 14 and 3 and between serotype 14 and serogroup 9, as we found two isolates (Bsp097 and Bsp098) were ST1569. ST1569 has been identified in serotypes 3,9A, and 9V. All the clinical serotype 14 isolates, expressed different levels of penicillin and ceftriaxone nonsusceptibility (Table 3 ), which is in accord with the data published recently [38]. This finding indicates that higher competence and plasticity of the genome likely afforded an advantage to pneumococcal strains to become more and more antimicrobial-resistant, and again supports that virulent clones might evolve to be more resistant in order to survive in the drug environment. Given the fact, to reduce the selective pressure, judicious use of antibiotics should never be overemphasized.

\section{Discussion}

A bacterial pathogen can be described by its "supragenome", which is composed of a "core genome" and an "distributed genome" [39-41]. In general, the core genome includes all genes responsible for the basic aspects of the biology of a species and its major phenotypic traits. In contrast, distributed genomes constitute to the species diversity and might encode supplementary biochemical pathways and functions not essential for bacterial growth but which confer selective advantages, such as adaptation to different niches and antimicrobial resistance. In this study, we added a complete genome to the pneumococcal supragenome. The analysis of distributed genome of CGSP14 indicates that pneumococcal supragenome is still open and evolving.

Table 3: Multilocus sequence typing and antimicrobial susceptibility of $S$. pneumoniae serotype 14 isolates

\begin{tabular}{|c|c|c|c|c|c|c|c|c|c|c|}
\hline \multirow[b]{2}{*}{ Strain } & \multirow[b]{2}{*}{ Sequence type (corresponding serotypes) } & \multicolumn{7}{|c|}{ MLST allelic profile } & \multicolumn{2}{|c|}{ MIC ( $\mu g / m l)$} \\
\hline & & $\operatorname{aroE}$ & $g d h$ & gki & recP & spi & $x p t$ & $d d l$ & Penicillin & Ceftriaxone \\
\hline CGSPI4 & $15(14)$ & I & 5 & 4 & 5 & 5 & 3 & 8 & 2 & I \\
\hline SPOI53 & $876(14)$ & 8 & 13 & 14 & 4 & 4 & 6 & 14 & 4 & 1 \\
\hline SPOI66 & $876(14)$ & 8 & 13 & 14 & 4 & 4 & 6 & 14 & 4 & 0.5 \\
\hline SP0286 & $876(14)$ & 8 & 13 & 14 & 4 & 4 & 6 & 14 & 8 & I \\
\hline SP0336 & $876(14)$ & 8 & 13 & 14 & 4 & 4 & 6 & 14 & 0.5 & 0.5 \\
\hline SP0340 & $876(14)$ & 8 & 13 & 14 & 4 & 4 & 6 & 14 & 4 & 2 \\
\hline SP0363 & $876(14)$ & 8 & 13 & 14 & 4 & 4 & 6 & 14 & 4 & I \\
\hline SP0386 & $876(14)$ & 8 & 13 & 14 & 4 & 4 & 6 & 14 & 4 & I \\
\hline SPO2I 10 & $13(14)$ & I & 5 & 4 & 5 & 5 & 27 & 8 & 8 & 2 \\
\hline SP0226 & $13(14)$ & 1 & 5 & 4 & 5 & 5 & 27 & 8 & 4 & 1 \\
\hline SP0233 & $13(14)$ & 1 & 5 & 4 & 5 & 5 & 27 & 8 & 4 & 1 \\
\hline SP0234 & $13(14)$ & 1 & 5 & 4 & 5 & 5 & 27 & 8 & 6 & 2 \\
\hline SP0282 & $13(14)$ & 1 & 5 & 4 & 5 & 5 & 27 & 8 & 4 & 1 \\
\hline Ssp0 017 & 46 (I4) & I & 5 & 4 & 16 & 31 & I & 14 & 0.5 & 8 \\
\hline Ssp097 & 46 (I4) & 1 & 5 & 4 & 16 & 31 & I & 14 & 0.5 & 0.25 \\
\hline SPOI7 & $46(14)$ & 1 & 5 & 4 & 16 & 31 & I & 14 & 0.5 & 0.5 \\
\hline SP044 & $46(14)$ & 1 & 5 & 4 & 16 & 31 & I & 14 & 8 & 2 \\
\hline SP033 & $46(14)$ & 1 & 5 & 4 & 16 & 31 & I & 14 & 8 & 8 \\
\hline Bsp097 & $1569(9 \mathrm{~V}, 3,9 \mathrm{~A})$ & 8 & II & 10 & 1 & 6 & 8 & 1 & 4 & 2 \\
\hline Bsp098 & $1569(9 \mathrm{~V}, 3,9 \mathrm{~A})$ & 8 & II & 10 & I & 6 & 8 & I & 4 & 4 \\
\hline SP0232 & $15(14)$ & 1 & 5 & 4 & 5 & 5 & 3 & 8 & 0.25 & 0.5 \\
\hline
\end{tabular}


Comparative analysis showed that gene communications occurred frequently among different strains of $S$. pneumoniae and among different species of the streptococcus genus by HGT, which has been demonstrated as a major force to drive bacterial evolution $[42,43]$. S. pneumoniae is able to efficiently acquire genetic materials from the large gene pool of the environment by means of transformation, transduction, and conjugation. We found that at the genome level, CGSP14 acquired at least eight foreign DNA elements from other organisms by HGT, as these blocks demonstrated deviation in the GC content. In sequence analysis, the genes encoded in these newly acquired elements may enhance the pathogenicity and antimicrobial resistance of CGSP14.

In CGSP14 genome, a remarkable feature is horizontal acquisition of two conjugative transposons and one genomic island. The three previously un-described mobile elements totally carried eight antimicrobial resistance genes, which promote the adaptation of organism to an environment with high drug pressure. Besides, most of the other accessory DNA elements were flanked by IS elements; for instance, the capsular locus, the predominant virulence determinant of $S$. pneumoniae, was a mobile genetic element flanked by IS elements. The dynamics of these mobile elements might attribute to the clinically important phenotypic shift in S. pneumonae.

Invasive pneumococcal disease cases due to serotypes included in the 7-valent vaccine continued to fall in the USA, but the overall invasive pneumococcal disease rate leveled off starting in 2002, largely due to an increase in cases caused by serotype 19A, which is not covered by the 7 -valent vaccine [44]. Given these trends, development of expanded-valency polysaccharide vaccines or ideally, a universal protein vaccine, is mandatory. It has been shown that in the case of $S$. agalactiae, the design of such vaccine was only possible using virulence-related dispensable genes [44]. To this end, sequencing of multiple genomes from $S$. pneumoniae to better probe the diversity of the pathogen and its pathogenic features is necessary and certainly will continue to surprise us with fascinating discoveries in the evolution of $S$. pneumoniae and impact on the clinical medicine.

\section{Conclusion}

Streptococcus pneumoniae serotype 14 is one of the most invasive among > 90 pneumococcal serotypes, often causing life-threatening invasive pneumococcal diseases in humans. S. pneumoniae has been evolving rapidly over time, resulting in a large amount of genetic diversity, despite the constraints imposed by the small genome size and complex genetic organization of the genome. In this study, we sequenced the entire genome of a serotype 14 clinical isolate (CGSP14), and carried out comprehensive comparison with other pneumococcal genomes. We found that the genome evolution of $S$. pneumoniae is driven largely by the host adaptations. In addition to horizontal gene transfer, recombination, which re-balanced the genome structure after events of gene loss or addition, also appears to be an important element in S. pneumoniae evolution. Human intervention in the form of mass vaccination and antimicrobial treatment reduced the burden of pneumococcal diseases, but has already accelerated the evolution of the pneumococcal genome. We conclude that such evolution results in a more virulent and antimicrobial-resistant $S$. pneumoniae serotype 14 .

\section{Methods \\ Bacterial strains}

Twenty-one S. pneumoniae serotype 14 isolates, including the sequenced CGSP14, were collected from patients with bacteremic pneumonia treated in Chang Gung Memorial Hospital and Children's Hospital, Taoyuan, Taiwan between 2004 and 2005. Serotyping was carried out by the quellung reaction with antisera from the Statens Serum Institut, Copenhagen, Denmark and verified by sequential multiplex PCR [45]. Minimum inhibitory concentrations (MICs) were determined by the E test (AB Biodisk, Solna, Sweden). CGSP14 is a serotype 14 clinical isolate derived from a child with necrotizing pneumonia, simultaneously complicated with HUS. The MICs of antimicrobial agents to CGSP14 are penicillin, $2 \mu \mathrm{g} / \mathrm{mL}$, erythromycin, $256 \mu \mathrm{g} / \mathrm{mL}$, and ceftriaxone, $1 \mu \mathrm{g} / \mathrm{mL}$.

\section{Multilocus sequence typing (MLST)}

The nucleotide sequences of 450-bp internal regions from the $a r o E, d d l, g d h, g k i, r e c P, s p i$, and $x p t$ genes were amplified by PCR using the primers described previously [46]. The gene fragments were sequenced on both strands, by using the same primers, on an ABI 3730 automated sequencer (Applied Biosystems, Foster City, USA). The sequences were then compared with those of the recognized alleles of each gene listed in the pneumococcal MLST website database http://spneumoniae.mlst.net by using BioEdit Sequence Alignment Editor. The web database http://www.mlst.net was used for assigning allele numbers for particular loci and the sequence type (ST) of each isolate was defined on the basis of the resulting allelic profiles.

\section{Whole genome sequencing}

The whole genome of $S$. pneumoniae CGSP14 was sequenced by using the random shotgun method. Three genomic libraries (1.5 2-kb inserts, 2 3-kb inserts and 4$\mathrm{kb}$ inserts) were constructed from randomly sheared genomic DNA. Random clones were sequenced using ETDye terminator chemistry, analyzed with an ABI 3700 sequencer (Applied Biosystems, Foster City, USA) and a MegaBACE 1000 sequencer (Amersham Biosciences, Swe- 
den). DNA sequences were analyzed and assembled using PHRED, PHRAP, and CONSED [47-49]. Gaps in the sequence were closed either by sequencing through primer-walking the plasmid templates or by direct sequencing of combinatorial PCR products. The completed genome contained 34,888 reads with an average length of $501 \mathrm{bp}$, resulting in 8 -fold sequence coverage. The complete genome sequence of CGSP14 has been deposited in the GenBank database with the accession number CP001033.

\section{Genome annotation}

Open reading frames (ORFs) were predicted by GLIMMER with the default parameters [50]. Putative ORFs shorter than 30 amino acids were eliminated and ORFs that overlapped were visually inspected, and removed as needed. The predicted ORFs were reviewed to define start codons on the basis of ribosomal-binding motifs and homologies. ORFs were further searched against the non-redundant protein database using BLAST [50]. Functional domains of putative proteins were identified by searching against Prosite, Blocks, and Pfam database http:// pfam.sanger.ac.uk/. Functional categories were assigned by searching all predicted proteins against COG database http://www.ncbi.nlm.nih.gov/COG. Transfer RNA genes (tRNAs) were identified using tRNAscan-SE [51], ribosomal RNA genes (rRNAs) and other structural RNAs were identified from BLAST similarity searches.

\section{Comparative genomic analysis}

Twelve penumococcal genome sequences and the proteincoding sequences per strain (R6: AE007317; D39: CP000410; TIGR4: AE005672; G54: CP001015; SP3BS71: AAZZ00000000; SP6-BS73: ABAA00000000; SP9BS68: ABAB00000000; SP11-BS70: ABAC00000000; SP14-BS69: ABAD00000000; SP18-BS74: ABA E00 0000 00; SP19-BS75: ABAF00000000 and SP23-BS72: AB AG 00000000 ) were obtained through the web site of NCBI http://www.ncbi.nlm.nih.gov. The remaining four genome sequences (Spn23F, SPnINV104B, SPnINV200, and SPnOXC141) were obtained through the web site of Sanger Institute http://www.sanger.ac.uk/, and the ORFs per strain were predicted by GLIMMER and annotated by searching against the nr database using BLAST. Each pair of the predicted ORFs among the seventeen penumococcal strains were aligned using BLASTP (E value $<10^{-5}$ and protein similarity $>40 \%$ ). The outputs were parsed by perl scripts written by ourselves to extract the orthologous genes shared among the seventeen penumococcal genomes. Alignment of the genomes of the five completely sequenced strains was accomplished with the MUMmer program [52]. ACT (Artemis Comparison Tool) was used to enable the visualization of BLAST comparisons between the genomes [53]. Single nucleotide polymorphism (SNP) analysis of each orthologous gene was accomplished using CLUSTALW [54].

\section{Abbreviations}

MLST: multilocus sequence typing; HGT: horizontal gene transfer; HUS: hemolytic uremic syndrome; CDS: proteincoding sequence; ORF: open reading frame; ST: sequence type.

\section{Competing interests}

The authors declare that they have no competing interests.

\section{Authors' contributions}

$\mathrm{CHC}, \mathrm{PT}$, and JY conceived and designed the experiments. $\mathrm{FD}, \mathrm{MHH}$, and $\mathrm{SH}$ performed the experiments. FD, PT, $\mathrm{PC}$, and $\mathrm{SH}$ analyzed the data. FD and $\mathrm{CHC}$ wrote the paper. All authors read and approved the final manuscript.

\section{Additional material}

\section{Additional file 1}

IS elements of the CGSP14 genome. The data show the list of the IS elements in the CGSP14 genome.

Click here for file

[http://www.biomedcentral.com/content/supplementary/14712164-10-158-S1.doc]

\section{Additional file 2}

Orthologues shared among the seventeen S. pneumoniae genomes. The data represent the core genes of the seventeen $\mathrm{S}$. pneumoniae genomes, assigned by their accession numbers in CGSP14

Click here for file

[http://www.biomedcentral.com/content/supplementary/14712164-10-158-S2.txt]

\section{Additional file 3}

COG assignment of the orthologous genes. The data show the alignment results by searching the core genes against COG database. Click here for file

[http://www.biomedcentral.com/content/supplementary/14712164-10-158-S3.txt]

\section{Additional file 4}

Comparison of antimicrobial resistance determinants among the seventeen S. pneumoniae genomes. The data show antimicrobial resistance determinants in the seventeen $\mathrm{S}$. pneumoniae genomes.

Click here for file

[http://www.biomedcentral.com/content/supplementary/1471-

2164-10-158-S4.xls]

\section{Additional file 5}

Capsule biosynthesis genes of CGSP14 and comparison of the capsular loci among three serotype 14 strains. This figure shows capsule biosynthesis genes of S. pneumoniae serotype 14 strains. Genes are represented by boxes colored according to the gene key, with gene designations above or below each box. Red bands indicate regions that are highly homologous between gene clusters.

Click here for file

[http://www.biomedcentral.com/content/supplementary/1471-

2164-10-158-S5.tiff] 


\section{Additional file 6}

Capsular biosynthesis pathway of S. pneumoniae CGSP14. This figure shows the capsule biosynthesis pathway of S. pneumoniae CGSP14. Click here for file

[http://www.biomedcentral.com/content/supplementary/14712164-10-158-S6.tiff]

\section{Additional file 7}

Putative surface proteins based on computer prediction. The data show the list of putative surface proteins of S. pneumoniae CGSP14.

Click here for file

[http://www.biomedcentral.com/content/supplementary/1471-

2164-10-158-S7.doc]

\section{Acknowledgements}

We thank Jianing Geng for helpful discussions. This work was supported by a grant from Chang Gung Memorial Hospital, Taoyuan, Taiwan (CMRPG46005I).

\section{References}

I. Klein DL: Pneumococcal disease and the role of conjugate vaccines. Microbial Drug Resist 1999, 5:147-157.

2. Doern GV, Brueggemann AB, Huynh H, Wingert E: Antimicrobial resistance with Streptococcus pneumoniae in the United States, 1997-98. Emerg Infect Dis 1999, 5:757-765.

3. Morita JY, Zell ER, Danila R, Farley MM, Hadler J, Harrison LH, Lefkowitz L, Reingold A, Kupronis BA, Schuchat A, et al.: Association between antimicrobial resistance among pneumococcal isolates and burden of invasive pneumococcal disease in the community. Clin Infect Dis 2002, 35:420-427.

4. Whitney CG, Farley MM, Hadler J, Harrison LH, Bennett NM, Lynfield $R$, Reingold A, Cieslak PR, Pilishvili T, Jackson D, et al.: Decline in invasive pneumococcal disease after the introduction of protein-polysaccharide conjugate vaccine. N Engl J Med 2003, 348: $1737-1746$.

5. Brueggemann $A B$, Pai R, Crook DW, Beall B: Vaccine escape recombinants emerge after pneumococcal vaccination in the United States. PLoS Pathogens 2007, 3:el68.

6. Waters AM, Kerecuk L, Luk D, Haq MR, Fitzpatrick MM, Gilbert RD, Inward C, Jones C, Pichon B, Reid C, et al.: Hemolytic uremic syndrome associated with invasive pneumococcal disease: the United kingdom experience. J Pediatr 2007, I 5 I: | 40- | 44.

7. Hsieh YC, Hsueh PR, Lu CY, Lee PI, Lee CY, Huang LM: Clinical manifestations and molecular epidemiology of necrotizing pneumonia and empyema caused by Streptococcus pneumoniae in children in Taiwan. Clin Infect Dis 2004, 38:830-835.

8. Lauderdale TL, Wagener MM, Lin HM, Huang IF, Lee WY, Hseih KS, Lai JF, Chiou CC: Serotype and antimicrobial resistance patterns of Streptococcus pneumoniae isolated from Taiwanese children: comparison of nasopharyngeal and clinical isolates. Diag Microbiol Infect Dis 2006, 56:421-426.

9. Gay K, Baughman W, Miller Y, Jackson D, Whitney CG, Schuchat A, Farley MM, Tenover F, Stephens DS: The emergence of Streptococcus pneumoniae resistant to macrolide antimicrobial agents: a 6-year population-based assessment. J Infect Dis 2000, 182:14|7-1424.

10. McKenzie H, Reid N, Dijkhuizen RS: Clinical and microbiological epidemiology of Streptococcus pneumoniae bacteraemia. J Med Microbiol 2000, 49:36I-366.

II. Mogdasy MC, Camou T, Fajardo C, Hortal M: Colonizing and invasive strains of Streptococcus pneumoniae in Uruguayan children: type distribution and patterns of antibiotic resistance. Pediatr Infect Dis J 1992, I I:648-652.

12. Croucher NJ, Walker D, Romero P, Lennard N, Paterson GK, Bason NC, Mitchell AM, Quail MA, Andrew PW, Parkhill J, et al:: The role of conjugative elements in the evolution of the multi-drug resistant pandemic clone Streptococcus pneumoniae Spain23F ST8I. J Bacteriol 2008, 191: | 480- 1489.
13. Hoskins J, Alborn WE Jr, Arnold J, Blaszczak LC, Burgett S, DeHoff BS, Estrem ST, Fritz L, Fu DJ, Fuller W, et al:: Genome of the bacterium Streptococcus pneumoniae strain R6. J Bacteriol 200I, I83:5709-57|7.

14. Lanie JA, Ng WL, Kazmierczak KM, Andrzejewski TM, Davidsen TM, Wayne KJ, Tettelin H, Glass JI, Winkler ME: Genome sequence of Avery's virulent serotype 2 strain D39 of Streptococcus pneumoniae and comparison with that of unencapsulated laboratory strain R6. J Bacteriol 2007, I89:38-5I.

15. Tettelin H, Nelson KE, Paulsen IT, Eisen JA, Read TD, Peterson S, Heidelberg J, DeBoy RT, Haft DH, Dodson RJ, et al.: Complete genome sequence of a virulent isolate of Streptococcus pneumoniae. Science 200I, 293:498-506.

16. Dopazo J, Mendoza A, Herrero J, Caldara F, Humbert Y, Friedli L, Guerrier M, Grand-Schenk E, Gandin C, de Francesco M, et al:: Annotated draft genomic sequence from a Streptococcus pneumoniae type I9F clinical isolate. Microbial Drug Resist 200I, 7:99-125.

17. Hiller NL, Janto B, Hogg JS, Boissy R, Yu S, Powell E, Keefe R, Ehrlich NE, Shen K, Hayes J, et al.: Comparative genomic analyses of seventeen Streptococcus pneumoniae strains: insights into the pneumococcal supragenome. J Bacteriol 2007, 189:8।86-8195.

18. Riley M: Functions of the gene products of Escherichia coli. Microbiological reviews 1993, 57:862-952.

19. Nakagawa I, Kurokawa K, Yamashita A, Nakata M, Tomiyasu Y, Okahashi N, Kawabata S, Yamazaki K, Shiba T, Yasunaga T, et al.: Genome sequence of an M3 strain of Streptococcus pyogenes reveals a large-scale genomic rearrangement in invasive strains and new insights into phage evolution. Genome Res 2003, I 3:1042-1055.

20. Ayoubi $P$, Kilic AO, Vijayakumar MN: Tn5253, the pneumococcal omega (cat tet) BM600 I element, is a composite structure of two conjugative transposons, Tn525I and Tn5252. J Bacteriol 1991, 173:1617-1622.

21. Kilic AO, Vijayakumar MN, al-Khaldi SF: Identification and nucleotide sequence analysis of a transfer-related region in the streptococcal conjugative transposon Tn5252. J Bacteriol 1994, 176:5। $45-5150$

22. Caillaud F, Carlier C, Courvalin P: Physical analysis of the conjugative shuttle transposon Tn / 545. Plasmid I987, I7:58-60.

23. Shaw JH, Clewell DB: Complete nucleotide sequence of macrolide-lincosamide-streptogramin B-resistance transposon Tn9I7 in Streptococcus faecalis. J Bacteriol 1985, 164:782-796.

24. Flannagan SE, Zitzow LA, Su YA, Clewell DB: Nucleotide sequence of the 18-kb conjugative transposon Tn9/6 from Enterococcus faecalis. Plasmid 1994, 32:350-354.

25. McDougal LK, Tenover FC, Lee LN, Rasheed JK, Patterson JE, Jorgensen JH, LeBlanc DJ: Detection of Tn9/7-like sequences within a Tn9/6-like conjugative transposon ( $T$ 3872) in erythromycin-resistant isolates of Streptococcus pneumoniae. Antimicrob Agents Chemother 1998, 42:231 2-23I8.

26. Grebe T, Hakenbeck R: Penicillin-binding proteins $2 b$ and $2 x$ of Streptococcus pneumoniae are primary resistance determinants for different classes of $\beta$-lactam antibiotics. Antimicrob Agents Chemother 1996, 40:829-834.

27. Brueggemann AB, Griffiths DT, Meats E, Peto T, Crook DW, Spratt $B$ : Clonal relationships between invasive and carriage Streptococcus pneumoniae and serotype- and clone-specific differences in invasive disease potential. J Infect Dis 2003, 187:| $1424-\mid 432$.

28. Brueggemann AB, Peto TE, Crook DW, Butler JC, Kristinsson KG, Spratt BG: Temporal and geographic stability of the serogroup-specific invasive disease potential of Streptococcus pneumoniae in children. J Infect Dis 2004, 190:1203-1211.

29. Song JH, Jung SI, Ko KS, Kim NY, Son JS, Chang HH, Ki HK, Oh WS, Suh JY, Peck KR, et al.: High prevalence of antimicrobial resistance among clinical Streptococcus pneumoniae isolates in Asia (an ANSORP study). Antimicrob Agents Chemother 2004, 48:2101-2I07.

30. Bentley S D, Aanensen D M, Mavroidi A, Saunders D, Rabbinowitsch E, Collins M, Donohoe K, Harris D, Murphy L, Quail M A, et al:: Genetic analysis of the capsular biosynthetic locus from all 90 pneumococcal serotypes. PLoS Genet 2006, 2:e31.

31. Kolkman MA, Wakarchuk W, Nuijten PJ, Zeijst BA van der: Capsular polysaccharide synthesis in Streptococcus pneumoniae serotype 14: molecular analysis of the complete cps locus 
and identification of genes encoding glycosyltransferases required for the biosynthesis of the tetrasaccharide subunit. Mol Microbiol 1997, 26:197-208.

32. Mitchell T]: The pathogenesis of streptococcal infections: from tooth decay to meningitis. Nat Rev Microbiol 2003, I :219-230.

33. Rosenow C, Ryan P, Weiser JN, Johnson S, Fontan P, Ortqvist A, Masure HR: Contribution of novel choline-binding proteins to adherence, colonization and immunogenicity of Streptococcus pneumoniae. Mol Microbiol 1997, 25:819-829.

34. Sanchez-Beato AR, Lopez R, Garcia JL: Molecular characterization of PcpA: a novel choline-binding protein of Streptococcus pneumoniae. FEMS Microbiol Lett 1998, I 64:207-2|4.

35. Lannelli F, Oggioni MR, Pozzi G: Allelic variation in the highly polymorphic locus pspC of Streptococcus pneumoniae. Gene 2002 284:63-7I.

36. Engelke G, Gutowski-Eckel Z, Hammelmann M, Entian KD: Biosynthesis of the lantibiotic nisin: genomic organization and membrane localization of the NisB protein. Appl Environ Microbiol 1992, 58:3730-3743.

37. Shen K, Gladitz J, Antalis P, Dice B, Janto B, Keefe R, Hayes J, Ahmed A, Dopico R, Ehrlich N, et al.: Characterization, distribution, and expression of novel genes among eight clinical isolates of Streptococcus pneumoniae. Infect Immun 2006, 74:321-330.

38. Forbes ML, Horsey E, Hiller NL, Buchinsky FJ, Hayes JD, Compliment JM, Hillman T, Ezzo S, Shen K, Keefe R, et al.: Strain-specific virulence phenotypes of Streptococcus pneumoniae assessed using the Chinchilla laniger model of otitis media. PLoS One 2008, 3:e1969.

39. Hogg JS, Hu FZ, Janto B, Boissy R, Hayes J, Keefe R, Post JC, Ehrlich GD: Characterization and modeling of the Haemophilus influenzae core and supragenomes based on the complete genomic sequences of Rd and I 2 clinical nontypeable strains. Genome Biol 2007, 8:RI03.

40. Medini D, Donati C, Tettelin H, Masignani V, Rappuoli R: The microbial pan-genome. Curr Opin Genet Dev 2005, I 5:589-594.

4I. Tettelin H, Masignani V, Cieslewicz MJ, Donati C, Medini D, Ward NL, Angiuoli SV, Crabtree J, Jones AL, Durkin AS, et al.: Genome analysis of multiple pathogenic isolates of Streptococcus agalactiae: implications for the microbial "pan-genome". Proc Nat Acad Sci USA 2005, 102:13950-13955.

42. Juhas M, Power PM, Harding RM, Ferguson DJ, Dimopoulou ID, Elamin AR, Mohd-Zain Z, Hood DW, Adegbola R, Erwin A, et al: Sequence and functional analyses of Haemophilus spp. genomic islands. Genome Biol 2007, 8:R237.

43. Marri PR, Hao W, Golding GB: Gene gain and gene loss in streptococcus: is it driven by habitat? Mol Biol Evol 2006 23:2379-2391.

44. Invasive pneumococcal disease in children 5 years after conjugate vaccine introduction - eight states, 1998-2005. MMWR Morb Mortal Wkly Rep 2008, 57:|44-|48.

45. Pai R, Gertz RE, Beall B: Sequential multiplex PCR approach for determining capsular serotypes of Streptococcus pneumoniae isolates. J Clin Microbiol 2006, 44: | 24- I3|

46. Enright MC, Spratt BG: A multilocus sequence typing scheme for Streptococcus pneumoniae: identification of clones associated with serious invasive disease. Microbiology 1998, | 44:3049-3060.

47. Ewing B, Green P: Base-calling of automated sequencer traces using phred. II. Error probabilities. Genome Res 1998, 8: $186-194$

48. Ewing B, Hillier L, WendI MC, Green P: Base-calling of automated sequencer traces using phred. I. Accuracy assessment. Genome Res 1998, 8: 175-185.

49. Gordon D, Abajian C, Green P: Consed: a graphical tool for sequence finishing. Genome Res 1998, 8:195-202.

50. Delcher AL, Harmon D, Kasif S, White O, Salzberg SL: Improved microbial gene identification with GLIMMER. Nucleic Acids Res 1999, 27:4636-4641.

51. Lowe TM, Eddy SR: tRNAscan-SE: a program for improved detection of transfer RNA genes in genomic sequence. Nucleic Acids Res 1997, 25:955-964.

52. Delcher AL, Kasif S, Fleischmann RD, Peterson J, White O, Salzberg SL: Alignment of whole genomes. Nucleic Acids Res 1999, 27:2369-2376.
53. Carver T], Rutherford KM, Berriman M, Rajandream MA, Barrell BG, Parkhill J: ACT: the Artemis Comparison Tool. Bioinformatics 2005, 2 I :3422-3423.

54. Thompson JD, Higgins DG, Gibson TJ: CLUSTAL W: improving the sensitivity of progressive multiple sequence alignment through sequence weighting, position-specific gap penalties and weight matrix choice. Nucleic Acids Res 1994, 22:4673-4680.
Publish with Biomed Central and every scientist can read your work free of charge

"BioMed Central will be the most significant development for disseminating the results of biomedical research in our lifetime. "

Sir Paul Nurse, Cancer Research UK

Your research papers will be:

- available free of charge to the entire biomedical community

- peer reviewed and published immediately upon acceptance

- cited in PubMed and archived on PubMed Central

- yours - you keep the copyright 